\title{
Vocal activity profile and dysphonia coping strategies in subjects with laryngeal cancer treated with radiotherapy
}

Michelle Ferreira Guimarães ${ }^{(1)}$ Letícia Osório Cézar de Oliveira(2) Elma Heitmann Mares Azevedo(1)

(1) Universidade Federal do Espírito Santo UFES, Vitória, Espírito Santo, Brasil.

(2) Laboratório de Deglutição e Voz (LaDVox) do Curso de Fonoaudiologia da Universidade Federal do Espírito Santo - UFES, Vitória, Espírito Santo, Brasil.

Study conducted at the Department of Speech and Hearing Therapy, Federal University of Espírito Santo - UFES, Vitória, Espírito Santo in association with the Radiotherapy sector of a private hospital, located in the city of Serra, Espírito Santo.

Conflict of interests: Nonexistent

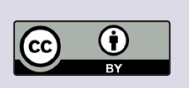

Received on: February 1, 2018

Accepted on: April 20, 2018

Mailing address:

Michelle Ferreira de Guimarães Av. Marechal Campos, 1498, Campus

Maruípe, prédio Básico II, sala 22

CEP: 29043-900 - Vitoria, Espírito Santo, Brasil

E-mail: guima.michelle@gmail.com

\section{ABSTRACT}

Purpose: to verify the vocal activity participation and the dysphonia coping strategies of subjects with laryngeal cancer treated with radiotherapy.

Methods: a cross-sectional trial involving 17 individuals with laryngeal cancer treated only by radiotherapy with vocal complaints after radiotherapy management. A sociodemographic questionnaire, the Brazilian versions of Voice Activity and Participation Profile and Voice Disability Coping Questionnaire protocols were applied.

Results: there was a prevalence of male participants $(n=16)$ and the mean age was 62 years. All were former smokers and 15 were former drinkers. Voice Activity and Participation Profile in mean scores was: overall,141.2, self-perception of vocal problem, 6.2, effect at work, 19.1, effects on daily communication, 60.5, effects on media, 20.1, and effects on their emotional, 36.7. In Voice Disability Coping Questionnaire mean scores were: overall, 71.7 , focusing on problem, 33, and focusing on emotion, 38.9.

Conclusion: the participants had high Voice Activity and Participation Profile scores as compared to the literature scores considered for dysphonic individuals in general, and activity with effects on daily communication was the most affected. Voice Disability Coping Questionnaire demonstrates that these patients adopt dysphonia coping strategies with greater focus on emotion.

Keywords: Laryngeal Neoplasms; Dysphonia; Adaptation, Psychological; Radiotherapy 


\section{INTRODUCTION}

The treatment modalities of the initial and advanced laryngeal neoplasm are surgery, radiotherapy, isolated or associated chemotherapy. Radiation oncology treatment aims to extinguish the tumor, reduce part of the tumor or reduce local pain to preserve the functions of breathing, swallowing and oral communication $^{1,2}$. On the other hand, radiotherapy can promote adverse effects to the patient of different degrees, such as fibrosis, chronic edema, laryngeal stenosis ${ }^{3}$, xerostomia, odynophagia, mucositis, dermatitis, osteoradionecrosis, trismus, caries and loss of taste ${ }^{4}$, resulting in increased or lower degree of dysphonia and different impacts on the patient's quality of life ${ }^{3}$.

In patients with laryngeal cancer undergoing radiotherapy, dysphonia may occur due to laryngeal edema, immobility, mucosal stiffness and/or vocal fold vibratory irregularity and laryngeal fibrosis, factors that restrict the necessary movement of cartilages and laryngeal muscles, modifying the glottic closure for phonation ${ }^{5}$. Such alterations can also lead to modifications in the physiology of oropharyngeal swallowing and conditions for the individual's overall well-being ${ }^{5,6}$.

Besides the functional issues, the treatment of laryngeal cancer causes changes in the activities of daily life of the patient with impact and impairment in primary functions for social interaction, in which the vocal function is highlighted $6-8$. Thus, many quality of life protocols verify the patient's self-perception of the impact of vocal changes in their lives and their analyses provide important information about the impact of difficulty in vocal production ${ }^{9}$.

The Vocal Activity Participation Profile (PPAV) protocol addresses the difference between the vocal problem limitation and the individual's willingness to participate in daily activities to better conduct the therapeutic process. Several protocols assess the impact of voice on quality of life. However, PPAV encompasses participation and limitation of activities, which are considerable factors in the World Health Organization's International Classification of Functioning, Disability, and Health. one of the only instruments that deals with aspects of work, social life, daily life and the expression of emotions, offering more details than the other validated protocols ${ }^{9}$.

Also, studies highlight the relevance of the inclusion of instruments focused on how the individual faces and deals with a problem. The Protocol of Strategies for Confronting Dysphonia (PEED-27) provides an understanding of how the individual handles dysphonia ${ }^{10}$.
Considering that there are no studies in the literature that address the impact of dysphonia in patients irradiated by laryngeal cancer regarding their participation in vocal activities and how they deal with this dysphonia, and that such data contribute strongly to the conduction of clinical speech and language rehabilitation, the aim of the study was to trace the profile of participation in vocal activities and to verify the coping strategies of the dysphonia of individuals irradiated by laryngeal cancer.

\section{METHODS}

This is a cross-sectional study approved by the Research Ethics Committee of the Health Sciences Center of the Federal University of Espírito Santo under opinion number 1,203,326.

There were 17 patients who received clarifications about the study and signed the Informed Consent Term. Patients treated with laryngeal cancer were treated with radiotherapy, complaining of dysphonia, between 40 and 70 years old, without cognitive alterations, capable of understanding and responding to the questions presented. Patients who did not complain of dysphonia, with neurological alterations, and/or cancer of the head and neck associated with laryngeal cancer were excluded.

The data were collected in the Radiotherapy sector of a private hospital in the city of Vitória, ES. Firstly, a sociodemographic questionnaire was applied with data regarding age, gender, time of onset of the disease, alcohol intake and use of tobacco, tumor staging and number of radiotherapy sessions they were submitted. Subsequently, the protocols of Participation Profile in Vocal Activities (PPAV) and the Protocol of Strategies of Confrontation of Dysphonia (PEED-27) were applied. The protocols were read and completed under the guidance of the researcher.

The PPAV protocol translated, adapted and validated into Brazilian Portuguese from the original Voice Activity and Participation Profile (VAPP) protocol is an instrument that considers the self-perception of vocal problem intensity, its effects at work, daily communication, social communication, and emotion. It consists of 28 questions distributed in five aspects with a specific score: self-perception of the intensity of the vocal problem, with a question and maximum score of 10 points; effects at work, with four questions and maximum score of 40 points; effects on daily communication, with twelve questions and maximum score of 120 points; effects in the social communication, with 
four questions and maximum score of 40 points; effects on emotion, with seven questions and maximum score of 70 points. The protocol still shows two additional scores, called Activity Limitation Score (PLA) and Participation Restriction Score (PRP), with maximum values of 100 points each. For the calculation of the PLA, a score was added on the ten peer questions that cover the aspects "work", "daily communication" and "social communication" (questions 2, 4, 6, 8, 10, 12, 14, 16, 18 and 20); for the PRP calculation was added the score of the ten odd questions of the same aspects mentioned above (questions 3, 5, 7, 9, 11, 13, $15,17,19$ and 21). The higher the score, the greater the limitation on vocal activities (difficulties imposed on activities) and the greater the restriction on participation (individual decreases or avoids participating in activities involving the use of voice). The maximum score of this protocol, adding up the scores of all questions is 280 points ${ }^{7,9}$.

The PEED-27 protocol translated and adapted culturally to Brazilian Portuguese from the VDCQ-27 (Voice Disability Coping Questionnaire-27) is a selfassessment tool, specifically developed to evaluate the way in which people with vocal problems face their voice problem ${ }^{13}$. It consists of 27 items categorized into two types of coping strategies: focused on the problem and focused on emotion. The strategies that focus on the problem (items: 2, 4, 7, 8, 11, 13, 14, 24, 25, 26) are the efforts used to modify the source of stress, and the strategies that focus on the emotion (items: 1, 3, 5, $6,9,10,12,15,16,17,18,19,20,21,22,23,27$ ) are attempts to regulate emotional stress caused by the stressor agent. The 27 items are evaluated on a 6-point Likert scale that analyzes the frequency of use of the strategy, with 0 (zero) corresponding to "never" and 5 to "always". The total score produced by the protocol can vary from 0 (zero) to 135 points, with zero indicating no strategy use and 135 for the use of all strategies described in the questionnaire ${ }^{7,8}$.

The sociodemographic data and the results obtained from the PPAV and PEED-27 were stored in a database using the Microsoft Excel 2007® program and analyzed by descriptive statistics.

\section{RESULTS}

There were 16 males and 1 female, with a mean age of 62 years old. They were all former smokers and most of them former alcoholics.

There was a prevalence of staging 3 and 4 . The number of radiotherapy sessions ranged from 10 to 35 .

The mean scores obtained with the PPAV protocol were: Total 141.2; self-perception of vocal problem 6.2; effects at work 19.1; effects on daily communication 60.5 ; effects on the social communication 20,1; effects on their emotion 36,7 (Table 1).

Table 1. Mean, minimum and maximum values of the scores obtained in the Profile of Participation in Vocal Activities (PPAV)

\begin{tabular}{|c|c|c|c|}
\hline PPAV & Mean & Minimum & Maximum \\
\hline Total Score & 141.5 & 29.0 & 242.0 \\
\hline Self-perception of the voice & 6.2 & 1.0 & 9.0 \\
\hline \multicolumn{4}{|l|}{ Effects on } \\
\hline Work & 19.1 & 5.0 & 36.0 \\
\hline Daily communication & 60.5 & 12.0 & 107.0 \\
\hline Social communication & 201 & 4.0 & 36.0 \\
\hline Emotion & 36.7 & 7.0 & 64.0 \\
\hline PLA & 50.7 & 11.0 & 87.0 \\
\hline PRP & 48.7 & 10.0 & 87.0 \\
\hline
\end{tabular}

Caption: PLA = Activity Limitation Score; PRP = Participation Restriction Score 
The mean scores obtained with the PEED protocol were: Total, 71.7 , focusing on the problem, 33, with focus on emotion, 38.9 (Table 2).

Table 2. Mean, minimum and maximum values of the scores obtained in the Protocol of Strategies of Confrontation in Dysphonia (PEED)

\begin{tabular}{|c|c|c|c|}
\hline PEED & Mean & Minimum & Maximum \\
\hline Total score & 71.7 & 27 & 125 \\
\hline \multicolumn{4}{|l|}{ Focus on } \\
\hline Problem & 33.0 & 10 & 78 \\
\hline Emotion & 38.9 & 9 & 66 \\
\hline
\end{tabular}

\section{DISCUSSION}

The effects of radiotherapy negatively influence vocal function, including roughness auditory perception, decrease in intensity, increase in phonatory tension and other persistent changes in vocal quality ${ }^{11,12}$. Radiation therapy for laryngeal cancer results in multidimensional vocal function deficits and these deficits notably persist in the long term. A recent study has shown that these multidimensional deficits have persisted for 2 to 7 years after finishing radiotherapy ${ }^{13}$.

In a study of perceptual-auditory and acoustic voice analysis with 15 patients submitted to radiotherapy and chemotherapy for laryngeal cancer, 33\% (five patients) presented adequate vocal quality or discrete dysphonia, $40 \%$ (six patients) presented moderate dysphonia and $27 \%$ (four patients) had severe dysphonia. On vocal quality after radiotherapy treatment for initial glottic tumor treatment, a study showed that $11 \%$ of the cases had normal voices, $44 \%$ had mild dysphonia, $27 \%$ to $28 \%$ had moderate dysphonia, and $16 \%$ to $17 \%$ had severe dysphonia ${ }^{14}$.

Laryngeal cancer affects men more frequently in the age group above 50 years old, data that corroborate with the findings of this study ${ }^{15,16}$. However, it should be noted that the prevalence being male does not exclude women and young people. The identification of many patients as former smokers $(100 \%)$ and former alcoholics $(88.2 \%)$ is in agreement with studies that associate tobacco and alcohol consumption as the most important and responsible risk factors for $90 \%$ of cases laryngeal cancer and oral cavity, with a risk up to 100 times higher in the case of combined use of the two substances ${ }^{17}$.

Voice self-perception protocols are an excellent method for identifying the individual's perception of the impact of voice disorders on their lives and in performing daily activities. The perception of the influence of dysphonia on the patient's life when self-referenced becomes a key point in therapeutic approaches since the patient's evaluation of a vocal alteration and the impact on daily life can influence motivation and adherence to treatment ${ }^{18}$.

For dysphonic patients, the following mean PPAV scores were considered: total of 98.8, self-perception of vocal problem with 5.5, effects on work with 13.3, effects on daily communication with 43.1, effects on social communication with 12.7 and effects on their emotion with $24,4^{9,19}$. It is observed that the data of this study obtained higher values than those mentioned (Table 1). It is worth mentioning that in the validation of PPAV for Brazilian Portuguese, the group with vocal complaints was constituted by patients with functional, organofunctional and organic dysphonia. In the "organic dysphonia group" the following mean scores were found: total of 73.3, self-perception of vocal problem with 6.13 , effects on work with 6.50 , effects on daily communication with 47.20 , effects on social communication with 6.17 and effects in the emotion $7.23^{\circ}$. When comparing these data with those of our study (Table 1), the only close score was related to self-perception of the vocal problem with 6.2, others are higher. The large difference in values between the two studies may be related to the fact that, in the validation study, patients with neurological dysphonia, endocrine, benign tumors or laryngeal carcinoma were included in the group "organic dysphonia", while in our study all patients have organic dysphonia due to laryngeal cancer and treated exclusively by radiation. It is also evidenced that the validation article of the PPAV does not mention how many patients had carcinoma, only refers that the group with vocal complaint was 
constituted by 25 participants, encompassing the three types of dysphonia.

Thus, no studies were found in the literature with application of this protocol only in patients with head and neck cancer. However, some studies evaluate the interference or impact of vocal problems, indirectly, on the life of patients undergoing treatment for laryngeal cancer with other instruments. In one study, 28 patients treated for laryngeal cancer were analyzed using the EORTC QLQ-C30 quality of life questionnaire. The authors state that survival is the result of the main interest of patients with a history of cancer, leading to a lower impact of vocal changes on quality of life ${ }^{20}$. Another study found that a group of patients treated for primary glottic cancer, either by radiotherapy or surgery via endoscopy, were satisfied with their speech and speech intelligibility, which did not prevent them from performing any daily life activities ${ }^{21}$.

In this study, the mean PLA score was 50.7 and the mean PRP score was 48.7. Considering that for this score the maximum score is $100^{9,19}$, it is observed that the patients presented median scores in relation to the limitations in the vocal activities and in relation to the restrictions of the participation of the vocal activities. These data corroborate the study that identified that the perception of a voice problem by the dysphonic subjects was positively correlated with the perception of limitation and restricted participation in the vocal activities. Forty patients with dysphonia and 40 patients without vocal alteration were studied, using vocal evaluation and PPAV. The group with dysphonia presented results above the mean considered for dysphonia in all parameters when compared to the group without vocal changes ${ }^{22}$.

In the validation study of PPAV, the score of the group "organic dysphonia" for PLA and PRP were 25.93 and 33.93 , respectively 9 . The values presented are lower than those found in this study.

One study evaluated 46 teachers with behavioralbased dysphonia and in the PPAV, they had mean scores of 87.8 , self-perception of voice of 4.9 , effects on work with 13.8 , effects on daily communication with 37,7 , effects on the social communication with 7.3 and effects on their emotion with 24,323. Comparing the literature, despite being distinct groups of participants, with our findings, the scores obtained were discrepant (Table 1), and our scores were higher in all aspects, showing that patients with laryngeal cancer after radiotherapy presented greater impact in the participation of vocal activities than teachers, for example.
The values found in the PPAV parameters in this study can be considered elevated when compared to the values cited in the literature for dysphonic patients in general and with dysphonia. It is believed that the patient suffering the impacts of laryngeal cancer and the radiation treatment, which contribute to the appearance of an organic dysphonia, tend to refer to a more intense vocal impact on factors that interfere in quality of life and communication when compared to individuals who present with functional or organofunctional dysphonia or other organic dysphonias not related to carcinoma.

Also, radiation sequels such as laryngeal edema, immobility, mucosal stiffness and/or vocal fold vibration irregularity, laryngeal fibrosis, and stiffness and tension throughout the cervical region may restrict aspects of vocal functionality and interfere directly with activities that require the use of voice, such as those investigated in the PPAV. Voice after radiotherapy treatment may not have sufficient potential and limit or even restrict oral communication of these patients, especially in daily and social activities.

An intriguing factor is that the mean age of the patients with the vocal complaint group of the PPAV validation study was 37 years old, and the "group with organic dysphonia" had a mean score of $6.5^{9}$, while in our study the mean age is 62 years old and for the same item the average score was 19.1. Considering that the Ministry of Health has been working on public policies aimed at active aging and that many older people remain working in Brazil, the vocal impact of the patients studied may begin to be more reported.

Voice disorders can have marked effects on the individual's life, which go beyond vocal impairment. However, there is little reference in current literature on how people deal with their voice problems ${ }^{24,25}$. Few studies stand out in the research of coping strategies specifically applied to voice disorders ${ }^{18}$.

Regarding PEED-27, a mean score of 51.86 was considered for patients in the general population with complaints of voice problems, and a mean score of 23.18 for those without a voice problem ${ }^{26}$. The analysis of this study presented data superior than the literature for the group with vocal complaint, whose average score was 71.7 .

Regarding the PEED-27 protocol, the most mentioned strategies were those focused on emotion and the most outstanding (82\%) were: "It is easier to deal with my voice problem when others are kind." Emotional coping strategies are characterized by the efforts of the individual to regulate their stressful 
emotions. Vaiano (2014) studied the coping strategies of dysphonia in patients with organic and behavioral dysphonia using PEED-10, a reduced version of PEED-27. The author observed that patients with organic dysphonia tend to use strategies with more focus on emotion, similar to the findings of this study ${ }^{27}$.

The use of this type of strategy allows the individual to modify his emotional state to reduce the unpleasant physical sensation from the state of stress ${ }^{24}$. Emotional coping strategies are also considered attempts to distort the reality that the individual is going through, as well as being a way of maintaining hope and optimism, avoiding facing the worst and reacting as if the problem had not due importance ${ }^{24,25}$.

Regarding strategies with a focus on the problem, the most mentioned were: "I find it easier to deal with my voice problem by understanding it better" (41\%) and "Resting the voice helps me deal with the voice problem" (35\%). These two strategies suggest that an individual with vocal disorders seeks more practical and concrete solutions to face his vocal changes ${ }^{25}$.

PEED-27 demonstrated that patients who underwent radiotherapy for laryngeal cancer treatment are more likely to use coping strategies for dysphonia with a focus on emotion. However, the predominance of one type of strategy by another is determined in part by the profile of each person, since some patients face their problems more actively than others ${ }^{26}$. In addition, studies report a higher occurrence of emotion-focused dysphonia coping strategies for patients with vocal complaints and benign lesions of the larynx, which corroborate with the findings in this study, despite the diagnosis of malignant lesion ${ }^{7,28}$.

Table 3. Answers of coping strategies of dysphonia focusing on the emotion and problem of the Coping Strategies Protocol in Dysphonia (PEED)

\begin{tabular}{lcc}
\hline Focus on Emotion & n & \% \\
\hline "It's easier to deal with my voice problem when others are kind" & 14 & 82.3 \\
"I wonder how good it would be to have no voice problem" & 4 & 23.5 \\
"I find it easier to deal with my voice problem by saying what I feel" & 6 & 35.2 \\
"I find it easier to deal with my voice problem by avoiding thinking about it" & 5 & 29.4 \\
"I keep to myself any concern about my voice problem" & 6 & 35.2 \\
"I think there's little I can do for my voice problem" & 5 & 29.4 \\
"Having a voice problem helped me to understand some important facts about my life" & 7 & 41.1 \\
"I find it easier to deal with my voice problem wishing it to end" & 5 & 29.4 \\
"I find it easier to deal with my voice problem making jokes about it" & 3 & 17.6 \\
"I try to accept my voice problem because there is nothing to be done" & 4 & 23.5 \\
"I think religion or prayer help me deal with my voice problem" & 5 & 29.4 \\
"I keep for myself the frustrations caused by my voice and few friends know what I feel" & 29.4 \\
"I try to convince myself that my voice problem does not hurt me so much" & 5 & 29.4 \\
"Having a voice problem has helped me be a better person" & 5 & 29.4 \\
"I ignore my voice problem by looking only at the good things in life" & 5 & 29.4 \\
"When my voice gets bad, I affect the others" & 5 & 23.5 \\
"I find it easier to deal with my voice problem when I compare myself to people with health problems worse & 4 & 6 \\
than mine" & 35.2 \\
\hline Focus on the Problem & & \\
\hline "I try to avoid situations that make my voice problem more evident" & 3 & 17.6 \\
"I try to get all possible information about my voice problem" & 5 & 29.4 \\
"Talking to friends and family about my voice problem helps me" & 3 & 17.6 \\
"I find it easier to deal with my voice problem by understanding it better" & 5 & 29.4 \\
"It's easier to get along with my voice problem when I do not speak" & 6 & 35.2 \\
"I find it easier to deal with my voice problem when I ask questions to the doctors" & 5 & 29.4 \\
"I find it easier to deal with my voice problem by avoiding to be with other people" & 4 & 23.5 \\
"Resting the voice helps me deal with the voice problem" & 6 & 35.2 \\
"I ask for help from others because of my voice problem" & 5 & 29.4 \\
"I try to do physical activities not to think about the voice" & 4 & 23.5 \\
\hline
\end{tabular}


It is believed that all the stigma of death risk surrounding cancer can help these patients to use more strategies to deal with dysphonia with a focus on emotion, using affective resources to deal with the restlessness, frustration, anxiety, and discouragement of the disease. In this sense, the application of PEED-27 may direct the speech-language pathologist to understand how the patient with laryngeal cancer deals with his voice problem, aware that the way he deals it, can directly influence the rehabilitation process.

Preliminary data are presented on participation in vocal activities and coping strategies of dysphonia in patients with laryngeal cancer treated exclusively by radiotherapy. Even with the great difficulty in grouping head and neck cancer patients treated with the same carcinoma in the same region and with the same treatment modality, future studies with a control group should be considered to obtain more robust results and analyzes.

\section{CONCLUSION}

Patients with laryngeal cancer and treated with exclusive radiotherapy have high PPAV scores when compared to the scores considered in the literature for dysphonic patients in general, and the activity with effects on daily communication is the most affected. PEED-27 demonstrates that these patients use coping strategies for dysphonia with a greater focus on emotion.

\section{REFERENCES}

1. Portas J, Socci PC, Scian PE, Queija SO, Ferreira AS, Dedivits RA et al. Swallowing after non-surgical treatment (radiation therapy/ radiochemotherapy protocol) of laryngeal cancer. Braz J Otorhinolaryngol. 2011;77(1):96-101.

2. Lôbo ALG, Martins GB. Consequências da radioterapia na região de cabeça e pescoço: uma revisão da literatura. Rev Port Estomatol Med Dent Cir Maxilofac. 2009;50(4):251-5.

3. Marciscano AL, Charu V, Starmer HM, Best SR, Quon $\mathrm{H}$, Hillel AT et al. Evaluating post-radiotherapy laryngeal function with laryngeal videostroboscopy in early stage glottic cancer. Front Oncol. 2017;7(124):1-9.

4. Campos RJDS, Leite ICG. Qualidade de vida e voz pós-radioterapia: repercussões para a fonoaudiologia. Rev. CEFAC. 2010;12(4):671-7.
5. Almeida FCS, Cazal C, Durazzio MD, Ferraz AR, Silva DP. Radioterapia em cabeça e pescoço: efeitos colaterais agudos e crônicos bucais. Rev Bras Patol Oral. 2004;3(2):62-9.

6. Prepageran N, Raman R. Delayed complication of radiotherapy: laryngeal fibrosis and bilateral vocal cord immobility. Med J Malaysia. 2005;60(3):377-8.

7. Oliveira G, Hirani SP, Epstein R, Yazigi L, Behlau M. Coping strategies in voice disorders of a brazilian population. J Voice. 2012;26(2):205-13.

8. Oliveira IB, Marialva DRS. Vocal handicap and coping strategy in dysphonia after laryngectomy. Audiol Commun Res. 2017;22:e1743.

9. Ricarte A, Oliveira G, Behlau M. Validation of the Voice Activity and Participation Profile Protocol in Brazil. CoDAS. 2013;25(3):242-9.

10. Oliveira G, Zambon F, Vaiano T, Costa F, Behlau M. Reduced versions of dysphonia coping protocols. CoDAS. 2016;28(6):828-32.

11. Bergstrom L, Ward EC, Finizia C. Voice rehabilitation for laryngeal cancer patients: Functional outcomes and patient perceptions. Laryngoscope. 2016;126(9):2029-35.

12. Sjögren EV, van Rossum MA, Langeveld TP, Voerman MS, van de Kamp VA, Friebel MO et al. Voice outcome in T1a midcord glottic carcinoma: laser surgery vs radiotherapy. Arch Otolaryngol Head Neck Surg. 2008;134(9):965-72.

13. Angadi V, Dressler E, Stemple J. Multidimensional study of vocal function following radiation therapy for laryngeal cancers. Annals of Otology, Rhinology \& Laryngology. 2017;126(6):483-92.

14. Caminero CMJ, Señaris GB, López LA, Núñez BF, Alonso PR, Suárez NC. Voice quality assessment after laryngeal cancer radiotherapeutic treatment at initial stages. Clin Transl Oncol. 2006;8(4):284-9.

15. Pimentel FL. Head and neck cancer: health related quality of life assessment considering clinical and epidemiological perspectives. Rev Bras Epidemiol. 2012;15(1):38-48.

16. Curado MP, Hashibe M. Recent changes in the epidemiology of head and neck cancer. Curr Opin Oncol. 2009;21(3):194-200.

17. Mehanna H, Jones TM, Gregoir EV, Angg KK. Oropharyngeal carcinoma related to human papillomavirus. British Medical Journal. 2010;340(7752):879-80.

18. Ferracciu CCS. Distúrbio de voz relacionado ao trabalho e estratégias de enfrentamento em professoras da rede pública estadual de Alagoas 
[Tese]. Rio de Janeiro (RJ): Escola Nacional de Saúde Pública Sergio Arouca; 2013.

19. Behlau M, Oliveira G, Santos LA, Ricarte A. Validação no Brasil de protocolos de auto-avaliação do impacto de uma disfonia. Pró- Fono R Atual. Cientif. 2009;21(4):326-32.

20. Finizia C, Hammerlid E, Westin T, Lindström J. Quality of life and voice in patients with laryngeal carcinoma: a post treatment comparison of laryngectomy (salvage surgery) versus radiotherapy. Laryngoscope.1998;108(10):1566-73.

21. Smith JC, Johnson JT, Cognetti DM, Landsittel DP, Gooding WE, Cano ER et al. Quality of life, functional outcome, and costs of early glottic cancer. Laryngoscope. 2003;113(1):68-7.

22. Ma EPM, Yiu EML. Voice activity and participation profile: assessing the impact of voice disorders on daily activities. $J$ Speech Lang Hear Res. 2001;44(3):511-24.

23. Tutya AS, Zambon F, Oliveira G, Behlau M. Comparação dos escores dos protocolos QVV, IDV e PPAV em professores. Rev Soc Bras Fonoaudiol. 2011;16(3):273-81.

24. Bretas MC. Análise comparativa da qualidade de vida e estratégias de enfrentamento de pacientes com disfonia decorrente ou não do câncer de laringe [Tese]. São Paulo (SP): Fundação Antônio Prudente; 2011.

25. Vivan AS, Argimon IIL. Coping strategies, functional difficulties, and associated factors in institutionalized elderly. Cad. Saúde Pública. 2009;25(2):436-44.

26. Oliveira GG. Estratégias de enfrentamento nos distúrbios de voz [Tese]. São Paulo (SP): Universidade Federal de São Paulo; 2009.

27. Vaiano TCG. Estratégias de enfrentamento nas disfonias orgânica e comportamental [Dissertação]. São Paulo (SP): Universidade Federal de São Paulo; 2014.

28. McHugh-Munier C, Scherer KR, Lehmann W, Scherer U. Coping strategies, personality, and voice quality in patients with vocal fold nodules and polyps. J Voice. 1997;11(4):452-61. 